\title{
MODELO PARA PLANEJAMENTO DA REDE REVERSA DE LATAS DE ALUMÍNIO PARA BEBIDAS ${ }^{1}$
}

\author{
Aura Maria Jalal ${ }^{a}$, Larissa Martins Henrique ${ }^{\text {a }}$, Eli Angela Vitor Toso ${ }^{\mathrm{a}^{*}}$ \\ aDepartamento de Engenharia de Produção Sorocaba \\ Universidade Federal de São Carlos - UFSCar, Sorocaba-SP, Brasil
}

Recebido 15/02/2017, aceito 01/09/2017

\begin{abstract}
RESUMO
O desempenho da reciclagem de latas de alumínio depende da estrutura da rede de logística reversa, pois a transferência de material dos pontos de geração até as plantas de fundição envolve altos custos de transporte. Uma estratégia para reduzir estes custos consiste em instalar centros de coleta próximos aos locais de descarte para receber, prensar as latas e unitizar a carga. Neste trabalho propomos um modelo de otimização para planejar a rede reversa, que considera decisões de localização e dimensionamento dos centros de coleta, alocação dos pontos de geração aos centros de coleta, e dimensionamento da frota de veículos. Testes usando dados da reciclagem no Brasil mostram que os custos fixos de instalação de centros de coleta são compensados pela redução nos custos de transporte, de fato, a abertura de novos centros de coleta implica em uma redução de $30 \%$ do custo total da rede.
\end{abstract}

Palavras-chave: Logística reversa, Planejamento de rede, Modelo de otimização.

\begin{abstract}
The environmental performance of aluminum cans recycling depends on the structure of the reverse logistics network, since the transfer of material from generation points to smelting centers involves high transportation costs. One strategy to reduce these costs consists in installing collection centers next to generation points to receive, press and load unitization. In this work, we propose an optimization model to plan the reverse network, considering decisions of locating and sizing collection centers, allocating generation points to collection centers, and sizing fleets. Tests using data from cans recycling in Brazil show that the fixed costs of installing collection centers offset high transport costs; in fact, installing collection centers implies a 30\% reduction in total costs.
\end{abstract}

Keywords: Reverse logistics, Network planning, Optimization model.

\footnotetext{
*Autor para correspondência. E-mail: eli@ufscar.br
}

DOI: 10.4322/PODes.2017.011 


\section{Introdução}

A reciclagem de alumínio no Brasil é uma importante atividade econômica que fatura cerca de $\mathrm{R} \$ 1,8$ bilhões por ano, somente a etapa de coleta de latas de alumínio injeta anualmente cerca de $\mathrm{R} \$ 730$ milhões na economia nacional (ABAL, 2016). Devido às suas propriedades, o alumínio pode ser usado em aplicações de alta qualidade, mesmo depois de ter sido reciclado várias vezes. Além dos benefícios econômicos, a reciclagem do alumínio tem como vantagens a redução da extração de matéria prima, a redução do descarte no meio ambiente e a economia de $95 \%$ de energia elétrica no processo de fabricação, em comparação com o processo a partir da matéria prima primária. Ainda, devido ao valor de mercado, a sucata de alumínio permite a geração de renda para milhares de famílias brasileiras envolvidas na etapa de coleta.

Os principais desafios da reciclagem estão relacionados com os altos custos da coleta seletiva e com a falta de sistemas adequadamente dimensionados quanto à capacidade de armazenamento e processamento de resíduos (Andrade e Ferreira, 2011). As redes de logística reversa são mais complexas devido à alta capilaridade dos pontos iniciais de coleta, que é maior no caso de países com grandes dimensões territoriais. O planejamento adequado das redes de logística reversa pode reduzir os custos de transporte e viabilizar a reinserção dos materiais na cadeia produtiva.

Para a configuração de redes logísticas, os modelos de localização de facilidades são importantes, pois permitem considerar simultaneamente vários aspectos dos problemas reais e apoiar o processo de planejamento da cadeia (Melo et al., 2009). O levantamento bibliográfico feito sobre modelos para configuração da rede de logística reversa apresenta muitos trabalhos, dos quais podemos destacar: Fleischmann et al. (1997); Zhou e Wang (2008); Pishvaee et al. (2010); Alumur et al. (2012); Kannan et al. (2012); e Ferri et al. (2015). Particularmente aplicado ao processo de reciclagem de alumínio, Logožar et al. (2006) propõem um modelo para reduzir os custos de transporte entre as várias unidades de uma planta industrial.

Este trabalho aborda a rede de logística reversa de latas de alumínio para bebidas no Brasil. Nos últimos 3 anos os investimentos para expansão desta rede reversa no Brasil somaram mais de $\mathrm{R} \$ 33$ milhões, com várias empresas abrindo novos centros de coleta em diferentes Estados. A abertura de centros de coleta próximos aos locais de consumo visa a redução dos custos de transporte, pois estes centros fazem a prensagem, unitização e consolidação das cargas. De acordo com empresas do setor, o planejamento da rede reversa de latas de alumínio para bebidas envolve decisões de localização de centros de coleta, de dimensionamento de capacidade dos mesmos, de dimensionamento da frota de veículos necessária em cada centro e decisões de transporte entre os pontos da rede.

$\mathrm{Na}$ literatura de planejamento de rede de logística reversa pesquisada, não foram encontrados modelos que considerassem as decisões de dimensionamento de frota de veículos. Neste contexto, propomos um modelo baseado no problema de localização de plantas com restrição de capacidade e distância (CDCPLP - Capacity and Distance Constrained Plant Location Problem) proposto por Albareda-Sambola et al. (2009). O modelo busca minimizar os custos fixos e variáveis associados ao transporte e os custos de instalação de centros de coleta, representando as decisões envolvidas no planejamento da rede reversa de latas de alumínio no Brasil.

Este artigo está organizado da seguinte forma, a próxima seção apresenta uma breve revisão bibliográfica sobre trabalhos que propõem modelos para apoiar as decisões de planejamento de redes de logística reversa. A seção 3 descreve a rede reversa de latas de alumínio para bebidas no Brasil. A seção 4 apresenta um modelo para o planejamento desta rede reversa, considerando suas particularidades. Na seção 5 são discutidos os resultados obtidos nos testes realizados com o modelo proposto. Finalmente a seção 6 discute as conclusões e perspectivas de pesquisa.

\section{Revisão Bibliográfica}

A logística reversa pós-consumo se inicia com a coleta de produtos descartados pelos consumidores finais e termina com o seu reparo, reuso, reciclagem, reprocessamento nos locais 
de remanufatura ou disposição final em locais adequados (Agrawal et al., 2016; Alshamsi e Diabat, 2015). Nos últimos anos as organizações têm dado maior importância à logística reversa devido ao aumento da preocupação com o meio ambiente, à construção de uma imagem junto aos consumidores e às imposições legais (Agrawal et al., 2016; Govindan et al., 2015; Zhou e Wang, 2008). Segundo Kannan et al. (2012), além dessas questões, a logística reversa também propicia benefícios financeiros ao retornar os produtos descartados para a cadeia produtiva, o que acaba fazendo com que as empresas adotem a logística reversa como uma ferramenta estratégica.

Comparando logística reversa com logística direta, há uma maior dificuldade no planejamento da logística reversa devido às incertezas na quantidade e qualidade dos produtos que retornam para o ciclo de produção (Niknejad e Petrovic, 2014). Outra característica que difere a logística reversa da direta é a importância dos centros de coleta/inspeção na configuração da rede (Pishvaee et al., 2010). Em geral, a quantidade desses centros de coleta/inspeção, sua localização, capacidades e tamanho são decisões estratégicas na configuração da rede de logística reversa. Alshamsi e Diabat (2015) consideram que a quantidade e localização desses centros tem um grande impacto nos custos de transporte da rede.

Muitos trabalhos na literatura propõem modelos matemáticos para apoiar as decisões de configuração de redes de logística reversa, sendo que a maioria propõe modelos gerais para a configuração de diferentes tipos de rede de logística reversa. Dentre esses autores estão Fleischmann et al. (1997) e Zhou e Wang (2008). O modelo proposto por Zhou e Wang (2008) é uma extensão do modelo de Fleischmann et al. (1997) pois considera consumidores, centros de distribuição, centros de recuperação de material e fábricas, ou seja, a estrutura do canal reverso é em três níveis, com três diferentes tipos de facilidades. A quantidade e a localização das fábricas, centros de distribuição e centros de recuperação de material são as variáveis de decisão do modelo.

A capacidade de processamento das plantas nas redes de logística reversa pode ser considerada como um parâmetro ou como uma decisão do problema. Pishvaee et al. (2010) propõem um modelo de programação linear inteira multiestágio que tem como objetivo minimizar tanto os custos de transporte quanto os custos de abertura das plantas e consideram a capacidade como um parâmetro. Já no modelo de Ferri et al. (2015), a capacidade é uma das decisões. Neste modelo os produtos são divididos em recuperáveis ou descartáveis, sendo que os primeiros são enviados para os centros de recuperação de material, enquanto os descartáveis são enviados para os centros de disposição final. Ferri et al. (2015) também abordam a inclusão das associações e cooperativas de catadores na configuração da rede reversa, apresentando um estudo de caso no Brasil. Este modelo procura incluir aspectos políticos e sociais junto com a minimização de custos da rede. Alumur et al. (2012) consideram capacidades iniciais e a possibilidade de expansão da capacidade das plantas existentes em um problema de configuração de rede de logística reversa multi-período. O horizonte de planejamento é finito e todas as decisões são implementadas no começo e no fim de cada período.

As decisões de localização podem incluir a consideração de questões ambientais. O modelo proposto por Kannan et al. (2012) combina as decisões de localização com a minimização dos impactos ambientais, particularmente a emissão de $\mathrm{CO} 2$. A função objetivo minimiza custos de coleta, disposição de material, transporte, custos fixos de abertura e custos de emissão em uma rede de logística reversa multiestágio. Os custos de transporte são considerados por unidade de produto transportado. Dekker et al. (2012) realizaram uma revisão dos modelos que integram as questões ambientais na logística indicando as diversas áreas que apresentam potencial de aplicação dos aspectos ambientais utilizando modelos matemáticos.

De forma geral, nos modelos para planejamento de redes logísticas, os custos de transporte são considerados apenas em função das distâncias envolvidas e das quantidades transportadas (custos variáveis). Particularmente neste aspecto, Albareda-Sambola et al. (2009) propõem um modelo para o problema de localização de plantas com restrição de capacidade e distância (CDCPLP- Capacity and Distance Constrained Plant Location Problem), que combina as decisões de localização com decisões de alocação de clientes e designação de veículos em cada planta. Estes autores consideram os custos fixos de transporte, associados à alocação de veículos em cada planta. 
Apesar da literatura sobre modelos para configuração de redes de logística reversa ser extensa, existem poucos trabalhos sobre a utilização dos modelos em contextos práticos.

\section{Logística Reversa de Latas de Alumínio para Bebidas}

A primeira etapa de desenvolvimento deste estudo consistiu na caracterização da rede de logística reversa de latas de alumínio para bebidas no Brasil, sua dinâmica de funcionamento e a identificação dos principais atores envolvidos. Para esta caracterização foram realizadas visitas a várias cooperativas de catadores, visitas a grandes empresas recicladoras de alumínio do Brasil, contatos com sucateiros e pesquisas em sites de associações do setor - Associação Brasileira do Alumínio (ABAL) e a Associação Brasileira dos Fabricantes de Latas de Alumínio (ABRALATAS). Como resultado, os atores da rede de logística reversa do alumínio pós-consumo no Brasil foram identificados, bem como suas relações institucionais. A Figura 1 esquematiza o ciclo fechado da rede logística de latas de alumínio para bebidas. Vale mencionar que o ciclo completo varia de 45 a 60 dias.

Figura 1: Rede logística de latas de alumínio para bebidas.

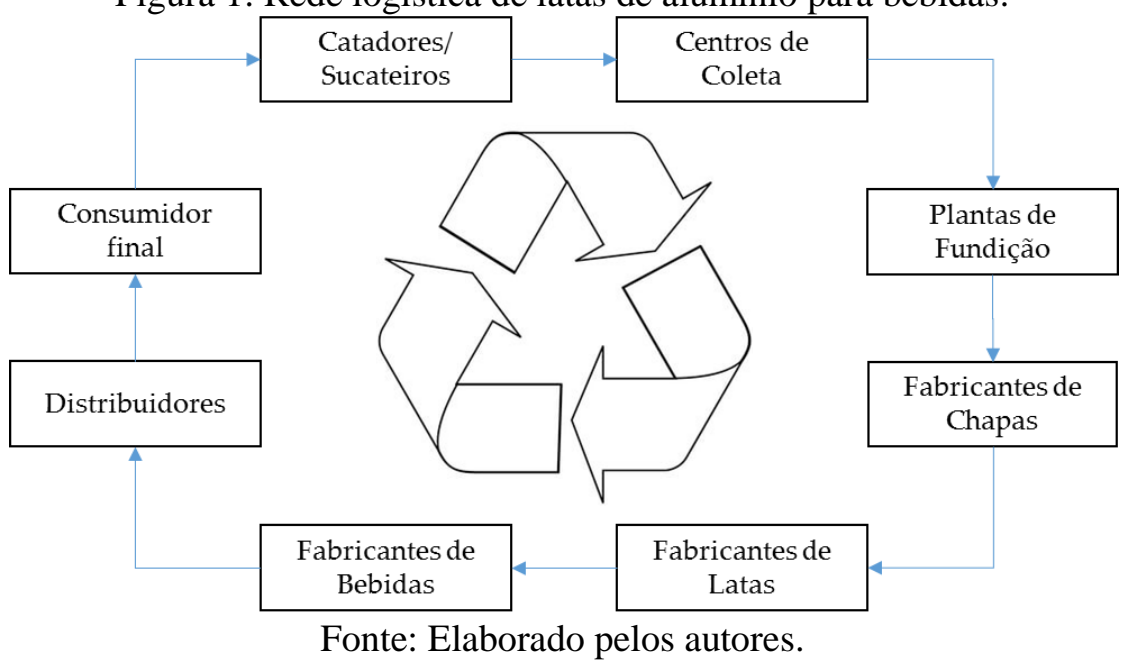

O ciclo reverso das latas de alumínio tem início após o consumo, onde o material descartado é coletado por catadores e sucateiros, que encaminham estes materiais para os centros de coleta. Como o valor de venda do material é alto quando comparado a outros resíduos, é frequente a presença de intermediários neste processo, principalmente em locais onde não existem centros de coleta que facilitem a venda direta para a indústria.

As empresas processadoras compram as latas de alumínio de catadores, cooperativas de catadores e sucateiros, utilizando uma estrutura própria de centros de coleta espalhados pelo país. Nesses centros de coleta é feito um pré-processamento, que consiste na triagem e compactação do material, que em seguida é enviado para as plantas de fundição e posteriormente para fabricantes de chapas de alumínio, que, por sua vez são a matéria-prima para a fabricação de latas de alumínio para bebidas.

Segundo a ABAL (2016), duas grandes empresas do setor de alumínio processam cerca de $70 \%$ de toda a sucata recuperada no Brasil. Estas empresas possuem 12 centros de coleta que atendem todas as regiões do Brasil e possuem plantas de fundição localizadas no Estado de São Paulo. Para desenvolvimento deste trabalho foram utilizados os dados das instalações destas duas empresas. De acordo com os dados coletados, em geral os centros de coleta são projetados para comprar e processar cerca de 500 toneladas de sucata por mês. Para facilitar o transporte e movimentação por empilhadeiras, nos centros de coleta as sucatas são transformadas em cargas unitizadas de acordo com um padrão exigido pelas empresas processadoras.

Em uma análise da configuração da rede reversa de latas de alumínio destacam-se os altos custos com o transporte do material até os centros de coleta, localizados em diversos estados do 
país, e dos centros de coleta até as plantas de fundição. Como mencionado anteriormente, nos últimos anos, as empresas recicladoras tem instalado novos centros de coleta para garantir o atendimento de suas demandas e reduzir os custos de transporte. Esta descentralização dos centros de coleta também pode trazer benefícios para os elos mais a montante da cadeia, onde estão os catadores e as cooperativas de reciclagem, que poderão comercializar o material diretamente com as recicladoras. Para a abertura destes centros de reciclagem é necessária a definição do número, local e tamanho das instalações, bem como a definição dos fluxos de material. Além disso, o dimensionamento da frota de veículos nos centros de coleta também foi apontado como um ponto importante para o planejamento desta rede, pois interfere significativamente na estrutura dos custos de transporte (fixos e variáveis) e na estrutura física da rede de coleta.

Desta forma, neste trabalho propomos um modelo de programação matemática que representa as decisões envolvidas na configuração desta rede: localização e dimensionamento de capacidade dos centros de coleta, alocação de regiões de geração de resíduos aos centros de coleta, alocação dos centros de coleta às unidades de processamento e dimensionamento da frota de veículos em cada centro de coleta.

\section{Modelagem do Problema}

Para a proposição de um modelo matemático que represente as decisões envolvidas no planejamento da rede de logística reversa de latas de alumínio para bebidas, foram feitas as seguintes considerações:

- O ciclo reverso é formado pelos seguintes pontos: locais de geração de resíduos, centros de coleta e plantas de fundição.

- A quantidade de material gerada é agregada em pontos de geração, que se considerou estarem localizados na capital de cada estado. Assumimos que esta quantidade é conhecida e que deve ser enviada para um ou mais centros de coleta.

- Os centros de coleta podem ser abertos com diferentes níveis de capacidade de processamento e, consequentemente, com diferentes custos de instalação. Os níveis de capacidade considerados são os mesmos para todos os centros, no entanto, os custos de instalação variam conforme o custo unitário por metro quadrado em cada local.

- As plantas de fundição possuem capacidade para processar todo o material coletado.

- Cada centro de coleta deve ter uma frota de veículos para coletar o material nos pontos de geração e para fazer a entrega do material pré-processado nas plantas de fundição.

- A frota de veículos em todos os centros de coleta é considerada homogênea e os custos fixos por veículo correspondem aos custos de depreciação, seguro e contratação de condutor e ajudante. Estes custos são incorridos tanto na opção por frota própria como na contratação de frete dedicado.

- Os custos variáveis de transporte são considerados por viagem e dependem da distância e do trecho percorrido. Desta forma, o custo de transporte até os centros de coleta é maior do que o custo de transporte a partir destes locais, pois o material é prensado e a carga consolidada.

Para formulação do modelo é utilizada a seguinte notação:

Conjuntos:

I pontos de geração de resíduos;

J locais candidatos à instalação dos centros de coleta;

$\mathrm{K}$ plantas de fundição de latas de alumínio;

$\mathrm{V}$ veículos alocados nos centros de coleta;

$\mathrm{S}$ níveis de capacidade dos centros de coleta (em toneladas). 
Parâmetros:

$l$ distância máxima que cada veículo pode percorrer no horizonte de tempo considerado;

$m$ número máximo de viagens por veículo no horizonte de tempo considerado;

$q$ capacidade de carga do veículo para carga não prensada (em toneladas);

$q$ ' capacidade de carga do veículo para carga prensada (em toneladas);

$b_{s} \quad$ capacidade de cada centro de coleta no nível $s$

$g_{i} \quad$ volume de resíduo gerado no estado $i$

a custo fixo por veículo (frota própria ou frete dedicado);

$f_{i s}$ custo fixo de instalação do centro de coleta no local $j$ com nível de capacidade $s$;

$d_{i j} \quad$ distância do estado $i$ ao centro de coleta $j$;

$d_{j k}^{\prime} \quad$ distância do centro de coleta $j$ ao centro de fundição $k$;

$c_{i j} \quad$ custo de cada viagem entre estado $i$ e o centro de coleta $j$;

$c_{j k}^{\prime} \quad$ custo de cada viagem entre o centro de coleta $j$ e o centro de fundição $k$.

Variáveis de decisão:

$x_{i j} \quad$ indica se o resíduo do estado $i$ é processado pelo centro $j\left(x_{i j}=1\right)$ ou não $\left(x_{i j s}=0\right)$;

$x_{j k}^{\prime} \quad$ quantidade transportada do centro $j$ para a planta $k$ (em toneladas);

$w_{j k v} \quad$ número de viagens do veículo $v$ do ponto $i$ ao centro de coleta $j$;

$w_{j k v}^{\prime} \quad$ número de viagens do veículo $v$ entre o centro $j$ e a planta de fundição $k$;

$y_{i s} \quad$ indica se o centro $j$ é aberto com nível de capacidade $s\left(y_{j s}=1\right)$ ou não $\left(y_{j s}=0\right)$;

$z_{j v} \quad$ indica se o veículo $v$ é utilizado pelo centro de coleta $j\left(z_{j k}=1\right)$ ou não $\left(z_{j k}=0\right)$.

Usando a notação acima o modelo matemático é formulado da seguinte maneira:

$\min \sum_{j \in J} \sum_{s \in S} f_{j s} y_{j s}+a \sum_{j \in J} \sum_{v \in V} z_{j v}+\sum_{i \in I} \sum_{j \in J} \sum_{v \in V} c_{i j} w_{i j v}+\sum_{j \in J} \sum_{k \in K} \sum_{v \in V} c_{j k}^{\prime} w_{j k v}^{\prime}$

Sujeito a:

$$
\begin{aligned}
& \sum_{j \in J} x_{i j}=1 \quad \forall i \in \mathrm{I} \\
& \sum_{i \in I} g_{i} x_{i j}=\sum_{k \in K} x_{j k}^{\prime} \quad \forall j \in \mathrm{J} \\
& \sum_{i \in I}^{i \in I} g_{i} x_{i j} \leq \sum_{s \in S}^{k \in K} b_{s} y_{j s} \quad \forall j \in \mathrm{J} \\
& \sum_{S \in S}^{i \in S} y_{j s} \leq 1 \quad \forall j \in \mathrm{J} \\
& \sum_{v \in V}^{s \in S} z_{j v} \leq|\mathrm{V}| \sum_{s \in S} y_{j s} \quad \forall j \in \mathrm{J} \\
& g_{i} x_{i j} \leq q w_{i j v} \quad \forall i \in \mathrm{I}, j \in \mathrm{J}, v \in \mathrm{V} \\
& x_{j k}^{\prime} \leq q^{\prime} w_{j k v}^{\prime} \quad \forall j \in \mathrm{J}, k \in \mathrm{K}, v \in \mathrm{V} \\
& \sum_{i \in I} w_{i j v}+\sum_{k \in K} w_{j k v}^{\prime} \leq m z_{j v} \quad \forall j \in \mathrm{J}, v \in \mathrm{V} \\
& \sum_{i \in I}\left(d_{i j}+d_{j i}\right) w_{i j v}+\sum_{k \in K}\left(d_{j k}^{\prime}+d_{k j}^{\prime}\right) w_{j k v}^{\prime} \leq l z_{j v} \quad \forall j \in \mathrm{J}, \quad v \in \mathrm{V} \\
& z_{j v} \leq z_{j, v-1} \\
& x_{i j}, y_{j s}, z_{j v} \in \mathrm{B} \\
& w_{i j v}, w_{j k v}^{\prime} \in \mathrm{Z}^{+} \\
& x_{j k}^{\prime} \in \mathrm{R}^{+} \\
& \forall j \in \mathrm{J}, \quad v \in \mathrm{V} /\{1\} \\
& \forall i \in \mathrm{I}, j \in \mathrm{J}, k \in \mathrm{K}, s \in \mathrm{S}, v \in \mathrm{V} \\
& \forall i \in \mathrm{I}, j \in \mathrm{J}, k \in \mathrm{K}, v \in \mathrm{V} \\
& \forall j \in \mathrm{J}, k \in \mathrm{K}
\end{aligned}
$$


A função objetivo (1) consiste em minimizar os custos totais, que são compostos pelos custos fixos de abertura de centros de coleta, custos fixos de veículos, custos de transporte de material não prensado dos pontos de geração aos centros de coleta e custos de transporte de material prensado dos centros de coleta aos centros de fundição. Note que o custo total de transporte é contabilizado em função do número de viagens necessárias, e o custo de cada viagem é incorrido mesmo que a capacidade do veículo não seja completamente utilizada.

A restrição (2) garante que todo resíduo gerado em cada ponto $i$ seja atendido por um centro de coleta $j$. A restrição (3) consiste no balanço de fluxo nos centros de coleta, garantindo que todo o material seja encaminhado para as plantas de fundição. A restrição (4) condiciona o envio de materiais à abertura de determinado centro de coleta $j$ e ao limite de capacidade definido pelo nível $s$, sendo que cada centro de coleta pode ser aberto com apenas um nível de capacidade, de acordo com a restrição (5). Já a restrição (6) condiciona a alocação de veículos à abertura dos centros de coleta, sendo que a cardinalidade do conjunto de veículos $(|\mathrm{V}|)$ correponde ao número máximo de veículos alocados em cada um.

As equações (7) e (8) relacionam a quantidade transportada ao número de viagens realizada por veículo, considerando a capacidade para transportar material não prensado (dos pontos de geração até os centros de coleta) e prensado (dos centros de coleta até as plantas de fundição), respectivamente. As restrições (9) e (10) limitam o número máximo de viagens e a distância máxima percorrida por veículo, respectivamente. Note que na restrição (10) são considerados os dois sentidos das viagens, pois os veículos devem sair e voltar para os centros de coleta. Estas restrições são importantes para dimensionar o total de veículos necessários em cada centro de coleta, uma vez que os custos fixos também incluem os custos com condutor e a lei do motorista (Lei $\mathrm{n}^{\circ}$ 13.103/2015) estabelece limites aos tempos de viagem. A restrição (11) ordena a alocação de veículos, por exemplo, o veículo 2 só será alocado a um centro $j$ se o veículo 1 foi alocado. Embora estas restrições não sejam necessárias para representação do problema, elas evitam simetrias entre as soluções, evitando múltiplas representações da mesma solução (AlbaredaSambola et al., 2009). Finalmente, as restrições (12) e (14) definem o domínio das variáveis de decisão.

\section{Testes com o Modelo}

Os experimentos para a validação do modelo proposto e avaliação dos resultados foram realizados utilizando a linguagem GAMS (Generic Algebraic Modeling System) versão 24.1.3, com o solver CPLEX versão 12.5, em um processador Intel Core i7, 16 GB de memória RAM e sistema operacional e Windows 7. Todos os testes foram executados com um limite de tempo de 3600 segundos, sendo que na maioria foram obtidos resultados ótimos em menos de 600 segundos.

\subsection{Descrição dos Dados}

Em relação aos parâmetros do problema, todos os dados foram estimados e calculados para um horizonte de planejamento de um ano. Os conjuntos I e $\mathbf{J}$ correspondem às capitais dos 27 estados brasileiros e as cidades onde atualmente estão instalados centros de coleta. A opção por considerar as capitais brasileiras como locais candidatos deve-se ao maior número de habitantes nessas cidades e às melhores condições de malha rodoviária. Da mesma forma, a consideração destes pontos como locais de concentração da geração de resíduos é baseada na maior densidade populacional, que pode ser observada no mapa da Figura 2 (regiões mais escuras). Para o conjunto $\mathrm{K}$ foram consideradas as plantas de fundição das duas maiores empresas do setor, que, por serem localizadas muito próximas umas às outras, foram agrupadas como um único local. Para o conjunto $\mathrm{V}$ admitiu-se que cada centro de coleta pode adquirir de 1 a 20 veículos. De acordo com informações obtidas em diferentes centros de coleta, para o conjunto $\mathrm{S}$ foram considerados 6 níveis de capacidade, a saber 1.000, 5.000, 10.000, 20.000, 50.000 e 10.0000 toneladas anuais, que correspondem a galpões de 50, 250, 500, 1.000, 2.500 e 5.000 metros quadrados, respectivamente. 
Figura 2: Comparação da densidade demográfica e a estimativa dos pontos de geração.

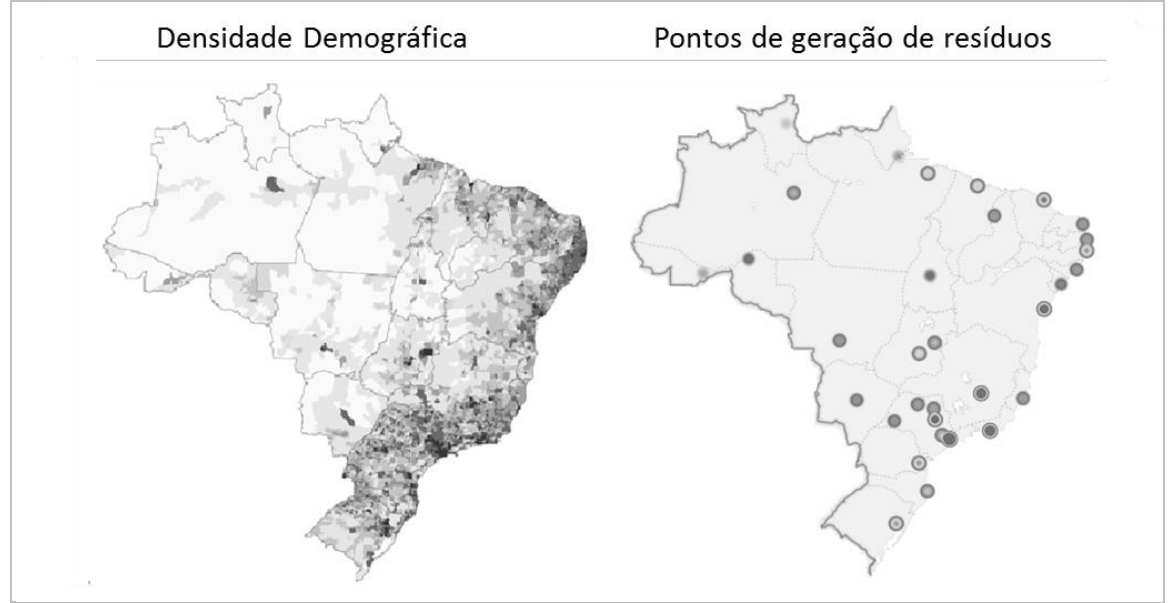

Fonte: Adaptado de IBGE (2017).

O volume de material a ser coletado em cada estado brasileiro $\left(g_{i}\right)$ foi calculado usando o percentual de participação de cada estado brasileiro na geração de resíduos sólidos do relatório Panorama dos Resíduos Sólidos no Brasil (ABRELPE, 2016), ou seja, distribuindo o volume total gerado pelo percentual de cada estado. Para calcular o volume total gerado, foi utilizada a informação de que $98,4 \%$ de toda a produção de latas de alumínio para bebidas é reciclada e também a informação de que $70 \%$ deste total é coletado pelas empresas consideradas na rede. Conforme mencionado, assumimos que o volume de resíduos a ser coletado concentra-se em um único ponto em cada estado, as suas respectivas capitais, e no estado de São Paulo em seis pontos adicionais à capital: Presidente Prudente, São José de Rio Preto, São Carlos, Sertãozinho, Itu e São Caetano do Sul. Esta é uma limitação do trabalho, pois a consideração de um número maior de cidades/pontos de geração representa os desafios do problema real com maior acuracidade.

O custo fixo de instalação por local candidato a centro de coleta e nível de capacidade $\left(f_{j s}\right)$ foi calculado com base no custo médio por metro quadrado em cada local candidato, obtidos do Sistema Nacional de Pesquisa de Custos e Índices da Construção Civil (SINAPI, 2015) e pelos valores de custo unitário básico em cada Estado (CUB/m2, 2016). Sendo que cada nível de capacidade corresponde à respectiva área de galpão $\left(50 \mathrm{~m}^{2} ; 250 \mathrm{~m}^{2} ; 500 \mathrm{~m}^{2} ; 1000 \mathrm{~m}^{2} ; 2500 \mathrm{~m}^{2}\right.$; $5000 \mathrm{~m}^{2}$ ). Os custos de equipamentos e maquinário não foram considerados, uma vez que estes são necessários para o processamento do material independentemente da localização dos centros de coleta. Ou seja, as fundições consideradas neste trabalho somente recebem latas prensadas com um padrão específico de enfardamento das latas de alumínio (formato e densidade - entre 400 e $550 \mathrm{~kg} / \mathrm{m}^{3}$ ), que tem como objetivo facilitar a movimentação por empilhadeiras.

O custo fixo por veículo (a) foi calculado a partir do custo anual com depreciação de um veículo com capacidade de 30 toneladas, custos de seguro e custo anual com contratação de condutor e ajudante. O valor obtido é de $\mathrm{R} \$ 131$ mil. Este valor foi comparado com o custo fixo de frete dedicado de alguns transportadores, validando-se a estimativa realizada. A distância máxima percorrida por veículo (l) foi estimada em $166.400 \mathrm{~km}$, considerando os limites de velocidade definidos no Artigo 61 do Código de Trânsito Brasileiro e o número de horas de trabalho em um ano. A capacidade do veículo para transportar as latas de alumínio não-prensadas $(q)$ e prensadas $\left(q^{\prime}\right)$ foi calculada com base na densidade das mesmas e nas dimensões do veículo. No caso das latas não prensadas (latas soltas ou de baixa prensa), a capacidade é de 16 toneladas por veículo, enquanto as prensadas superam o limite máximo do veículo, de 30 toneladas, portanto, considerou-se esse valor.

As distâncias entre os pontos de geração e os locais candidatos a pontos de coleta $\left(d_{i j}\right)$ e entre os locais de coleta e as plantas de fundição $\left(d_{j k}^{\prime}\right)$ foram calculadas utilizando o Google Maps. Os custos de transporte em cada viagem $\left(c_{i j}\right.$ e $c_{j k}^{\prime}$ ) foram calculados com base nas distâncias e inclui despesas com combustível, pedágios, manutenção, pneus, entre outros custos variáveis, 
conforme dados obtidos junto a transportadores. Os custos de transporte não consideram de forma explícita o número de toneladas transportadas e, portanto, a diferença entre carga prensada e nãoprensada. No entanto, o número de viagens $\left(w_{j k v}\right.$ e $\left.w_{j k v}^{\prime}\right)$ depende da capacidade do veículo, assim, o número de viagens necessárias para transportar o material não prensado (16 toneladas) é maior do que o número de viagens para transportar o material prensado (30 toneladas). Ou seja, o custo para transportar o material prensado é menor.

\subsection{Resultados}

A aplicação dos dados acima descritos no modelo matemático (1)-(14) resulta na abertura de 31 dos 33 locais candidatos a centros de coleta. A Figura 3 apresenta os resultados do modelo em relação à localização e dimensionamento da capacidade dos centros. Note que, a maioria dos centros é aberta com níveis de capacidade baixos, sendo que 3 foram abertos com capacidade de processamento de 1000 toneladas/ano; $16 \mathrm{com}$ capacidade de 5000 toneladas/ano; $7 \mathrm{com}$ capacidade de 10 mil toneladas/ano; 4 com capacidade de 20 mil toneladas/ano. O centro de coleta localizado na cidade de São Paulo foi aberto com uma capacidade muito alta, de $50 \mathrm{mil}$ toneladas/ano, o que já era esperado, pois o estado de São Paulo é responsável pela geração cerca de $28 \%$ da quantidade total de resíduos de latas de alumínio do Brasil. Apenas os centros de São Caetano do Sul e Maceió não são abertos.

Figura 3: Resultado para localização e capacidade dos centros de coleta.

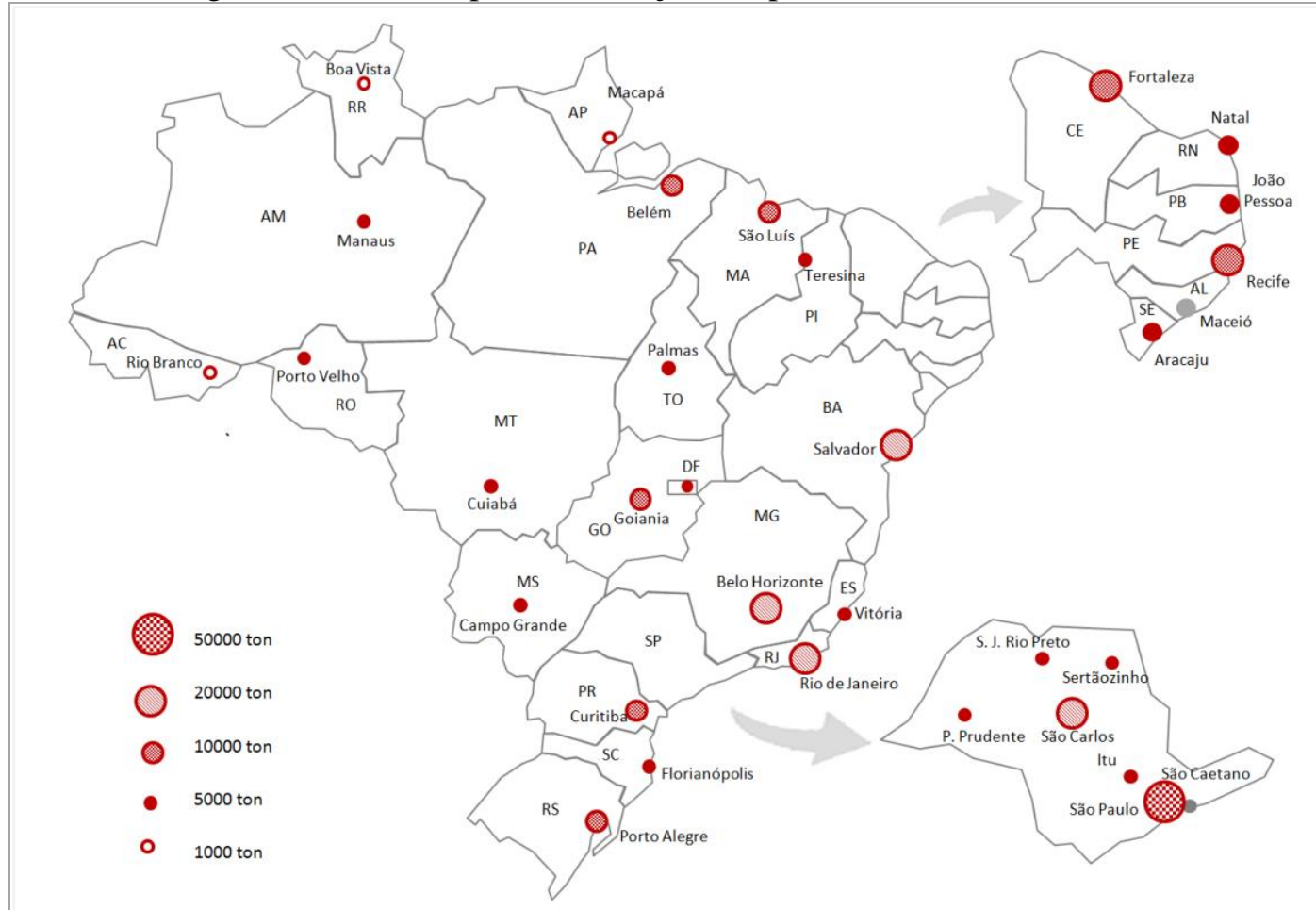

Fonte: Elaborado pelos autores.

Para transportar todo o resíduo de latas de alumínio é necessária uma frota de 95 veículos nos 31 centros de coleta. A taxa de utilização dos veículos é bastante alta, sendo que um mesmo veículo tanto pode ser utilizado para coleta nos pontos de geração quanto para envio do material prensado para as plantas de fundição. Como em quase todos os pontos de geração são instalados centros de coleta, o número de viagens para coleta é de 344 viagens, que correspondem à coleta e envio nos trechos, São Caetano do Sul - São Paulo e Maceió-Aracaju, utilizando apenas três veículos. No entanto, o número total de viagens entre os centros de coleta e as plantas de fundição é de 5.977, o que requer a utilização de 95 veículos. 
A Tabela 1 apresenta os resultados detalhados para cada centro de coleta. Podemos observar que o número de veículos designados a cada centro de coleta depende da quantidade de material processado e da distância até o centro de fundição $\left(d_{j k}^{\prime}\right)$, que determinam o total de viagens necessárias, de acordo com as restrições de capacidade dos veículos (8) e de distância máxima a ser percorrida (10). Por exemplo, note que os centros em Cuiabá e Florianópolis são abertos com a mesma capacidade (5000 toneladas/ano) e mesmo número de veículos alocados (2), sendo que em Cuiabá o total coletado é bem menor. Isso ocorre porque a distância de Cuiabá até a planta de fundição é muito maior, portanto, um único veículo atingiria o limite de distância máxima permitida.

Tabela 1: Resultados de dimensionamento da capacidade dos centros e da frota.

\begin{tabular}{|l|c|c|c|c|}
\hline \multicolumn{1}{|c|}{ Centro de coleta } & $\begin{array}{c}\text { Capacidade } \\
\text { (toneladas/ano) }\end{array}$ & $\begin{array}{c}\text { Distância até } \\
\text { fundição }(\mathbf{K m})\end{array}$ & $\begin{array}{c}\text { Total coletado } \\
\text { (toneladas) }\end{array}$ & \#Veículos \\
\hline Boa Vista & 1.000 & 4.751 & 332 & 1 \\
\hline Macapá & 1.000 & 3.397 & 552 & 1 \\
\hline Rio Branco & 1.000 & 3.621 & 499 & 1 \\
\hline Manaus & 5.000 & 4.002 & 3.444 & 6 \\
\hline Aracaju & 5.000 & 2.093 & 4.077 & 4 \\
\hline João Pessoa & 5.000 & 2.698 & 2.912 & 4 \\
\hline Teresina & 5.000 & 2.801 & 2.696 & 4 \\
\hline Natal & 5.000 & 2.864 & 2.500 & 3 \\
\hline Brasília & 5.000 & 1.124 & 3.756 & 2 \\
\hline Campo Grande & 5.000 & 1.149 & 2.166 & 2 \\
\hline Cuiabá & 5.000 & 1.644 & 2.637 & 2 \\
\hline Florianópolis & 5.000 & 843 & 4.078 & 2 \\
\hline Porto Velho & 5.000 & 3.114 & 1.185 & 2 \\
\hline Itu & 5.000 & 242 & 4.007 & 1 \\
\hline Palmas & 5.000 & 1.852 & 1.025 & 1 \\
\hline Presidente Prudente & 5.000 & 697 & 2.297 & 1 \\
\hline São Jose RP & 5.000 & 555 & 2.754 & 1 \\
\hline Sertãozinho & 5.000 & 462 & 3.007 & 1 \\
\hline Vitória & 5.000 & 801 & 2.734 & 1 \\
\hline Fortaleza & 10.000 & 2.853 & 8.069 & 10 \\
\hline Recife & 10.000 & 2.586 & 7.337 & 8 \\
\hline São Luís & 10.000 & 3.082 & 5.990 & 8 \\
\hline Belém & 10.000 & 2.011 & 5.770 & 5 \\
\hline Porto Alegre & 10.000 & 1.283 & 7.181 & 4 \\
\hline Goiânia & 10.000 & 1.053 & 5.518 & 3 \\
\hline Curitiba & 10.000 & 556 & 7.291 & 2 \\
\hline Salvador & 20.000 & 1.897 & 12.267 & 10 \\
\hline São Carlos & 20.000 & 365 & 10.589 & 2 \\
\hline Belo Horizonte & 20.000 & 513 & 15.755 & 1 \\
\hline Rio Janeiro & 20.000 & 293 & 18.141 & 1 \\
\hline São Paulo & 50.000 & 148 & 28.315 & 1 \\
\hline & $500:$ & & \\
\hline
\end{tabular}

Fonte: Elaborado pelos autores.

A rede proposta pelo modelo resulta em uma solução ótima com um custo de $\mathrm{R} \$ 36,6$ milhões para coletar uma quantidade de aproximadamente 180 mil toneladas/ano, indicando a abertura de 31 dos 33 locais candidatos a centros de coleta. Deste total, os custos de instalação dos centros correspondem apenas a 7\% do total, enquanto os custos fixos de transporte correspondem a $34 \%$. Em relação aos custos variáveis de transporte, como a rede ficou bastante descentralizada, apenas $1 \%$ do total corresponde aos custos de transporte de material não prensado. Já os custos de transporte de material prensado correspondem a 58\% dos custos totais, 
o que era esperado, uma vez que os centros de coleta estão descentralizados e as plantas de fundição concentram-se em um único local. A Tabela 2 apresenta os resultados obtidos para os custos anuais da rede de coleta proposta pelo modelo.

Tabela 2: Custos totais resultantes do modelo.

\begin{tabular}{|l|r|c|}
\hline \multicolumn{1}{|c|}{ Termos da Função Objetivo } & \multicolumn{1}{c|}{ Custo (R\$) } & Percentual (\%) \\
\hline Custos de Instalação de Centros de Coleta & 2.615 .121 & $7 \%$ \\
\hline Custos Fixos de Veículos & 12.475 .827 & $34 \%$ \\
\hline Custos de Transporte Material Não Prensado & 331.635 & $1 \%$ \\
\hline Custos de Transporte Material Prensado & 21.118 .519 & $58 \%$ \\
\hline Custo Total & 36.541 .101 & $100 \%$ \\
\hline
\end{tabular}

Fonte: Elaborado pelos autores.

Estes resultados da proporção entre os custos corroboram a estratégia das empresas de expandir o número de centros de coleta. É importante ressaltar que neste estudo, consideramos que os custos fixos de instalação correspondem apenas aos custos de manter um galpão, os custos de equipamentos, por exemplo, não foram considerados, pois as empresas visitadas recebem apenas o material já enfardado de forma específica para facilitar o manuseio. Atualmente, todo o material coletado é processado nos centros de coleta já existentes. Ou seja, assumimos que os custos fixos relacionado ao processamento independem do número, nível de capacidade e localização dos centros de coleta.

Uma consideração importante para a proposição do modelo para a rede de logística reversa de latas de alumínio é a integração das decisões de dimensionamento da frota de veículos em cada centro com as decisões de localização e dimensionamento da capacidade dos mesmos. Particularmente quando os custos fixos para alocação de veículos, seja por frota própria ou contratação de frete dedicado, são muito altos. Para testar o impacto desta integração foi realizado um teste resolvendo o modelo sem o dimensionamento de frota, ou seja, sem as restrições (7)(11). Os valores obtidos para as variáveis de localização $\left(y_{j s}\right)$ e de fluxo $\left(x_{i j}, x_{j e}^{\prime}\right)$ foram fixados e o modelo (1)-(14) foi resolvido novamente. Denominamos o modelo original como integrado e a abordagem testada como abordagem hierárquica, ou seja, aonde as decisões mais estratégicas de localização e alocação precedem as decisões mais táticas de dimensionamento de frotas. Os resultados destes testes são apresentados na tabela 3.

Tabela 3: Comparação dos resultados do modelo com uma abordagem hierárquica.

\begin{tabular}{|l|r|r|}
\hline & Abordagem integrada & Abordagem hierárquica \\
\hline \# centros de coleta & 31 & 33 \\
\hline \# veículos & 95 & 98 \\
\hline Total de viagens - não prensado & 344 & 0 \\
\hline Total de viagens material prensado & 5.977 & 5.979 \\
\hline Custos de Instalação & 2.615 .121 & 2.708 .480 \\
\hline Custos Fixos de transporte & 12.475 .827 & 12.869 .800 \\
\hline Custos de Transporte Material Não Prensado & 331.635 & 221.448 \\
\hline Custos de Transporte Material Prensado & 21.118 .519 & 20.892 .589 \\
\hline Custo Total & 36.541 .101 & 36.692 .318 \\
\hline
\end{tabular}

Fonte: Elaborado pelos autores.

Note na Tabela 3 que o número de centros de coleta abertos e o número total de veículos são diferentes nas abordagens, resultando em custo total maior da abordagem hierárquica. Vale ressaltar que o resultado da localização de alocação na abordagem hierárquica é muito dependente da estimativa dos custos de transporte, pois não considera a estrutura em termos de custos fixos e variáveis. Ainda, em termos de desempenho computacional, com o conjunto de dados testado, a solução ótima é alcançada em cerca de 480 segundos na abordagem integrada e na abordagem integrada cada etapa foi resolvida em cerca de 60 segundos. 


\subsection{Comparação com a Rede Atual}

A coleta de dados junto às empresas consideradas neste trabalho indicou 12 centros de coleta já instalados, embora já existissem planos de aumento deste número. Para comparar os resultados da rede proposta pelo modelo com a rede existente foram feitos dois testes:

- Teste A: Fixa a localização dos centros de coleta atuais, o modelo decide o nível de capacidade, os fluxos e o número de veículos necessário.

- Teste B: Fixa o número de centros de coleta atuais, o modelo decide quais abrir, o nível de capacidade, os fluxos e o número de veículos necessário.

Como o número de centros instalados na rede no momento da coleta de dados era de apenas 12 pontos, os testes A e B resultaram na abertura de centros de coleta com capacidade maior. No teste B, somente 4 dos locais escolhidos para a instalação dos centros de coleta correspondem aos locais aonde já existem centros (São Paulo, Goiânia, Salvador e Recife), o que indica oportunidades de melhoria na rede reversa. A Tabela 4 apresenta os resultados destes dois testes e sua comparação com a rede proposta no teste inicial.

Tabela 4: Comparação dos resultados dos testes A e B com a rede proposta pelo modelo.

\begin{tabular}{|l|r|r|r|}
\hline & Modelo & Teste A & Teste B \\
\hline \# centros de coleta & 31 & 12 & 12 \\
\hline \# veículos & 95 & 125 & 95 \\
\hline Total de viagens material não prensado & 344 & 6.221 & 3.490 \\
\hline Total de viagens material prensado & 5.977 & 5.968 & 5.968 \\
\hline Custos de Instalação de Centros de Coleta & 2.615 .121 & 2.435 .533 & 1.942 .966 \\
\hline Custos Fixos de Veículos & 12.475 .827 & 16.415 .561 & 12.475 .827 \\
\hline Custos de Transporte Material Não Prensado & $331.635,1$ & 16.347 .843 & 7.083 .512 \\
\hline Custos de Transporte Material Prensado & 21.118 .519 & 17.153 .605 & 19.504 .567 \\
\hline Custo Total & 36.541 .101 & 52.352 .542 & 41.006 .871 \\
\hline
\end{tabular}

Fonte: Elaborado pelos autores.

$\mathrm{O}$ teste A resulta no maior valor de custo total, $\mathrm{R} \$ 52.352 .542$, sendo que os custos fixos de transporte aumentam em $31,5 \%$, pois é necessária uma frota de 125 veículos. O teste $\mathrm{B}$, que mantém o número de centros de coleta, mas permite a avaliação de outros pontos de instalação em relação aos atuais, resultando em um custo total de $\mathrm{R} \$ 41.006 .871$. Ou seja, apenas a realocação dos centros existentes geraria uma redução de $21,67 \%$ nos custos totais.

Note na Tabela 3 que o custo fixo de instalação de centros de coleta nos três testes tem valores próximos, variando entre $\mathrm{R} \$ 1,95$ e $\mathrm{R} \$ 2,62$ milhões. Este resultado mostra que a descentralização dos pontos de coleta não implica em um aumento substancial nos custos de instalação. Além disso, os custos de instalação representam somente $1 \%$ do custo total. Por outro lado, os custos de transporte, fixos e variáveis, são significativamente alterados nos diferentes testes, sendo que os custos de transporte de material não prensado variam de $\mathrm{R} \$ 332$ mil, no caso da rede com 31 pontos de coleta, e mais de $\mathrm{R} \$ 16$ milhões, no caso da rede com apenas 12 pontos de coleta, nos locais definidos pelas empresas (teste A).

Entre os custos parciais, apenas os custos variáveis de transporte de material prensado (dos centros de coleta até as plantas de fundição) são significativamente maiores no teste inicial. Isso ocorre porque o fluxo de material prensado enviado é muito maior, já que o custo total do transporte de latas de alumínio prensado é menor, devido à maior densidade da carga.

Informações mais recentes obtidas nos sites das empresas estudadas mostram que as mesmas estão investindo na descentralização dos pontos de coleta. No entanto, ainda existe uma concentração grande de pontos de coleta no estado de São Paulo, que é o maior gerador de resíduos de latas de alumínio para bebidas. Vale mencionar que a descentralização dos centros de coleta permite que catadores e sucateiros possam vender as latas de alumínio coletadas diretamente para as empresas recicladoras, obtendo maior lucro na venda. 


\subsection{Testes com Variação de Parâmetros}

\subsubsection{Quantidade de Resíduos Gerada $\left(g_{i}\right)$}

Os testes anteriores foram realizados considerando a informação fornecida pelas empresas de reciclagem visitadas, que processam $70 \%$ de todo o resíduo de latas de alumínio para bebidas gerado no Brasil. No entanto, segundo dados da ABAL, em 2015 o Brasil reciclou 97.9\% das embalagens produzidas, permanecendo como líder na reciclagem de latas de alumínio desde 2001.

Além do mais, dados da produção de latas de alumínio para bebidas no Brasil apontam um crescimento acumulado de 100,6\% nos últimos 10 anos (ABRALATAS, 2016). Desta forma, para avaliar o impacto do aumento da geração de resíduos na configuração da rede reversa, foram realizados os seguintes testes:

- Teste C: Aumentando a quantidade de resíduos para $100 \%$ do total reciclado.

- Teste D: Aumentar a quantidade de resíduos para $100 \%$ do total produzido.

A Figura 4 apresenta uma comparação entre os resultados destes testes. Note que o aumento da quantidade de material nos pontos de geração aumenta os custos totais da rede reversa, principalmente pelo aumento de custos de transporte (fixos e variáveis). Os centros abertos no teste inicial são mantidos no teste (C), exceto Rio Branco e Macapá, que tinham sido abertos em capacidade muito baixa, e Brasília que tinha sido aberto com capacidade baixa. São abertos centros de coleta em Maceió e São Caetano do Sul; no teste (D) são abertos os mesmos centros de coleta que o teste inicial, exceto Rio Branco e Boa Vista; sendo aberto o centro de São Caetano do Sul. Os custos de instalação aumentam com a abertura dos novos centros e também pelo aumento da capacidade de centros já abertos. Estes resultados indicam que a rede formada pelos pontos de coleta é resiliente a mudanças na quantidade de resíduos gerados, sendo indicado que os gestores estejam atentos para a possibilidade de projetar locais com potencial para ampliação de capacidade (Cardoso et al., 2015).

Figura 4: Comparativo dos resultados dos testes (C) e (D) com os resultados iniciais (teste A).

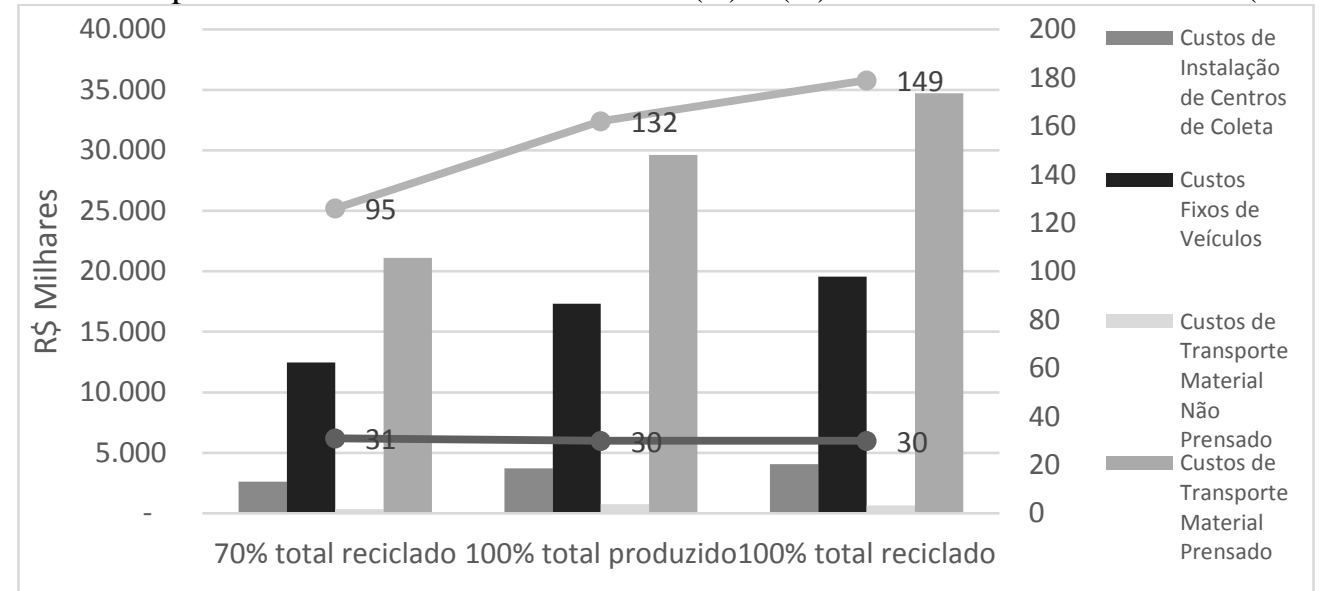

Fonte: Elaborado pelos autores.

Vale ressaltar que o aumento na quantidade de resíduos nestes testes não considerou alterações de distribuição na geração entre os estados, ou seja, o aumento em todos os pontos de geração foi proporcional às quantidades estimadas inicialmente.

\subsubsection{Alteração nos Custos $\left(f_{j s}, a, c_{i j} e c_{j k}^{\prime}\right)$}

Com o objetivo de avaliar o desempenho do modelo proposto frente à variação dos componentes de custos da função objetivo foram realizados os seguintes testes:

- Teste E: Redução do custo fixo de instalação $\left(f_{j s}\right)$ para $80 \%$ do valor estimado inicialmente. 
- Teste F: Aumento do custo fixo de instalação $\left(f_{j s}\right)$ para $120 \%$ do valor inicial.

- Teste G: Redução do custo fixo de veículo (a) para $80 \%$ do valor estimado inicialmente.

- Teste H: Aumento do custo fixo de veículo (a) para $120 \%$ do valor inicial.

- Teste I: Redução dos custos de transporte $\left(c_{i j} e c_{j k}^{\prime}\right)$ para $80 \%$ do valor inicial.

- Teste J: Aumento dos custos de transporte $\left(c_{i j} e c_{j k}^{\prime}\right)$ para $120 \%$ do valor inicial.

A Figura 5 apresenta os resultados obtidos nestes testes. Note que a variação nos custos fixos de instalação, $0,8^{*} f$ e $1,2 * f$, que correspondem aos testes $(\mathrm{E})$ e $(\mathrm{F})$, respectivamente, não impacta significativamente nos custos totais. Isso pode ser explicado pelo fato da porcentagem dos custos totais de instalação de centros de coleta corresponder a somente $1 \%$ dos custos totais.

$\mathrm{O}$ custo total da rede reversa mostra-se mais sensível em relação à variação nos custos de transportes (fixos e variáveis). A variação nos custos fixos de transporte, $0,8^{*} a$ e $1,2^{*} a$, que correspondem aos testes $(\mathrm{G}) \mathrm{e}(\mathrm{H})$, respectivamente, impacta o custo total de forma proporcional. A redução deste custo em $20 \%$ resulta em uma redução de $7 \%$ nos custos totais, e, da mesma forma, o aumento em $20 \%$ resulta em um aumento de $6,5 \%$ no total. Da mesma forma, o teste com redução de $20 \%$ no custo de transporte variável, teste (I), resulta em uma redução de $12 \%$ no custo total, enquanto que o teste com aumento de $20 \%$, teste (J), resulta em um aumento de $11 \%$.

Figura 5: Resultados dos testes com variação nos parâmetros de custo.

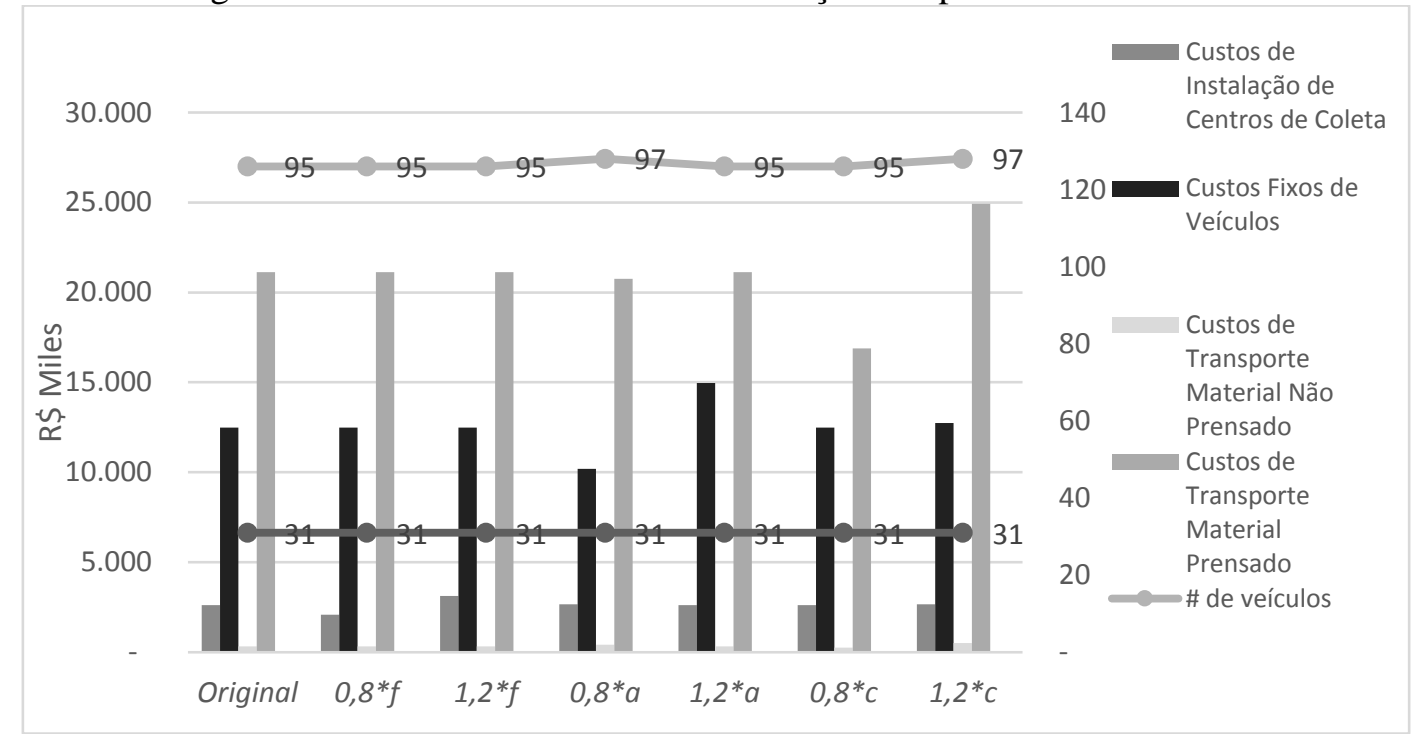

Fonte: Elaborado pelos autores.

O resultado destes testes mostra que a variação nos custos não implica em alterações no número total e na localização dos centros de coleta. Estes resultados também parecem indicar a resiliência da rede reversa proposta pelo modelo em função de variações no mercado de reciclagem de latas de alumínio para bebidas.

Ainda, em relação à estrutura de custos, supondo que os custos fixos de instalação tenham sido subestimados, realizamos testes aumentando significativamente esse custo. Mesmo considerando os custos fixos como o dobro dos custos estimados, os resultados indicam a abertura de 29 centros de coleta e um total de 104 veículos para o transporte.

\subsection{Testes com Total de Centros de Coleta Limitado}

Além da relação entre custos fixos e variáveis, é comum nos problemas de planejamento de redes logísticas, principalmente em problemas de localização, a consideração de um limite para o número de instalações a serem abertas, pois os valores para investimento podem não estar disponíveis imediatamente. Especificamente no caso da rede de latas de alumínio para bebidas, 
vale ressaltar que os custos totais da rede são distribuídos entre os diferentes agentes, além das empresas de reciclagem, por exemplo, sucateiros (Figura 1). E, neste caso, estamos considerando que o investimento em centros de coleta é feito pelas empresas recicladoras. Desta forma, foram realizados testes limitando o número de centros de coleta a serem abertos. Os resultados destes testes são apresentados na Tabela 5.

Tabela 5: Comparação dos testes com diferente número de centros.

\begin{tabular}{|l|r|r|r|r|r|r|}
\hline & $\mathbf{1 5}$ centros & $\mathbf{1 8}$ centros & $\mathbf{2 1}$ centros & $\mathbf{2 4}$ centros & $\mathbf{2 7}$ centros & $\mathbf{3 0}$ centros \\
\hline \#centros abertos & 15 & 18 & 21 & 24 & 27 & 30 \\
\hline \# veículos & 93 & 93 & 94 & 95 & 94 & 95 \\
\hline $\begin{array}{l}\text { \# viagens } \\
\text { não-prensado }\end{array}$ & 2.473 & 1.898 & 1.636 & 1.093 & 683 & 365 \\
\hline \#viagens prensado & 5.969 & 5.970 & 5.971 & 5.973 & 5.976 & 5.976 \\
\hline $\begin{array}{l}\text { Custos de } \\
\text { instalação }\end{array}$ & 2.089 .750 & 2.222 .396 & 2.360 .619 & 2.459 .361 & 2.537 .789 & 2.605 .056 \\
\hline $\begin{array}{l}\text { Custos fixos } \\
\text { transporte }\end{array}$ & 12.213 .178 & 12.213 .178 & 12.344 .502 & 12.475 .827 & 12.344 .502 & 12.475 .827 \\
\hline $\begin{array}{l}\text { Custos transporte } \\
\text { não-prensado }\end{array}$ & 4.992 .956 & 3.790 .635 & 3.056 .416 & 1.974 .687 & 1.036 .750 & 436.846 \\
\hline $\begin{array}{l}\text { Custos de } \\
\text { transporte prensado }\end{array}$ & 20.067 .480 & 20.183 .521 & 19.883 .265 & 20.262 .519 & 20.853 .177 & 21.068 .368 \\
\hline Custo Total & 39.363 .363 & 38.409 .730 & 37.644 .802 & 37.172 .393 & 36.772 .218 & 36.586 .097 \\
\hline
\end{tabular}

Fonte: Elaborado pelos autores.

Conforme esperado, os resultados mostram que o número de centros abertos sempre é o maior valor permitido, pois os custos de transporte do material não-prensado são significativamente maiores que os custos dos materiais prensados. Um resultado interessante nestes testes foi o impacto da abertura de centros de coleta sobre os custos de instalação e de transporte de material não-prensado, conforme mostra a Figura 5.

Figura 5: Variação dos custos de instalação e transporte de material não prensado.

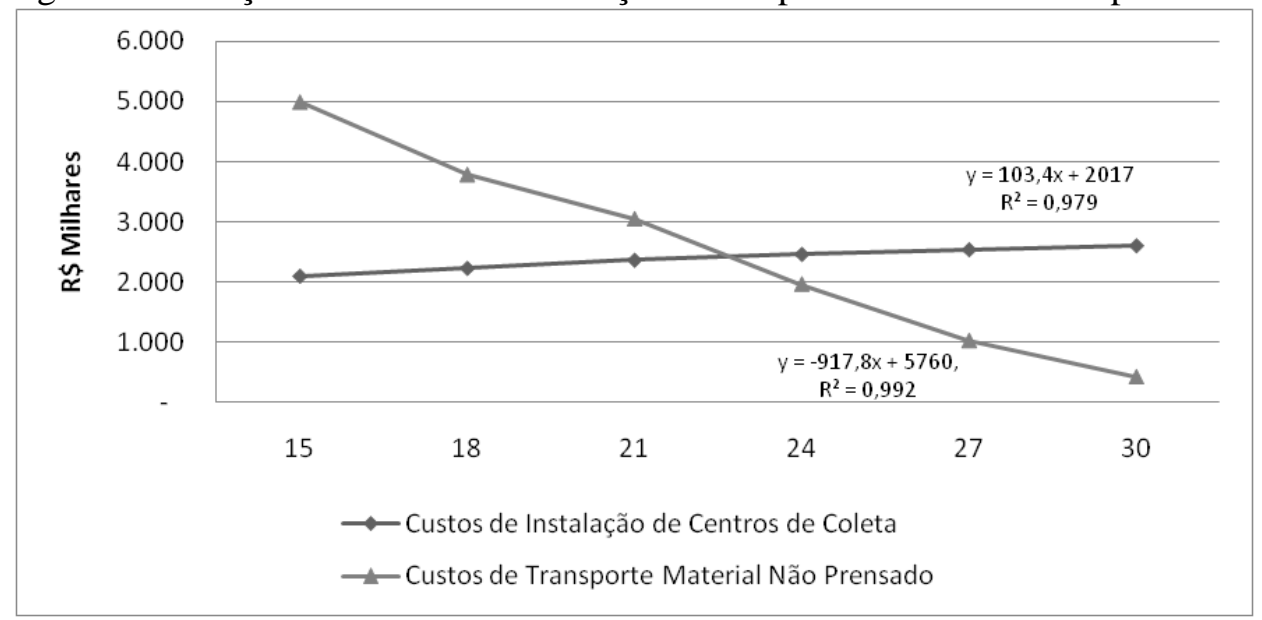

Fonte: Elaborado pelos autores.

Ajustando a uma linha reta, temos que a taxa de crescimento no custo de instalação é menor que a taxa de decrescimento dos custos de transporte de material não prensado, o que comprova que o aumento nos custos de instalação é compensado pela redução dos custos de transporte de material não prensado, reduzindo o custo total da rede.

Além da vantagem em relação aos custos de transporte, observe na Tabela 5 que o número total de viagens para transporte do material (prensado e não-prensado) com limite de 15 centros abertos é de 8442 viagens, e, quando o limite é de 30 centros, este valor é reduzido para 6341 
viagens. Ou seja, um maior número de centros de coleta implica em uma redução de $25 \%$ do total de viagens, o significa do ponto de vista ambiental uma redução na emissão de gases poluentes, como o gás carbônico $\left(\mathrm{CO}_{2}\right)$.

\section{Conclusões}

Este trabalho aborda a rede de logística reversa de latas de alumínio para bebidas no Brasil. Para apoiar as decisões de planejamento nesta rede propomos um modelo matemático que considera as decisões de localização de centros de coleta de resíduos, o dimensionamento da frota de veículos nestes centros e os fluxos de material dos pontos de geração até os centros de coleta e destes até as plantas de fundição. O objetivo é reduzir os custos fixos e variáveis de transporte e os custos fixos de instalação dos centros de coleta.

Foram feitos testes com dados da rede de duas empresas que são responsáveis por $70 \%$ de toda a reciclagem de latas de alumínio do Brasil. Os resultados evidenciam a necessidade de descentralização dos pontos de coleta, que são responsáveis pela triagem e compactação do material. A descentralização, com a abertura de centros de coleta em quase todos os estados do país, reduz significativamente os custos totais de transporte, e, consequentemente reduz os custos totais, visto que os custos variáveis de transporte correspondem a quase $60 \%$ do custo total da rede de coleta.

Para comparar a rede proposta pelo modelo com a rede das empresas estudadas, foram feitos testes fixando a localização dos centros de coleta existentes e também fixando o número de centros de coleta. Desta forma, é possível avaliar os resultados com base nos mesmos parâmetros do modelo. Os custos da rede proposta pelo modelo podem ser reduzidos em $30 \%$ em relação à rede com os centros existentes mantidos nos mesmos locais, e em $21,7 \%$ em relação a uma rede com o mesmo número de centros de coleta, mas com a possibilidade de realocação destes pontos.

O alumínio é uma comoditie com contratos futuros e de opções negociados por Bolsas de Mercadorias como a de Nova Iorque (NYMEX), a Bolsa de Mercadorias de Tóquio (TOCOM) e a Bolsa de Mercadorias Metálicas de Londres (LME). Este mercado está sujeito a variações econômicas que afetam tanto os preços do material de fontes primárias quanto o material reciclado. Para avaliar a resiliência da rede proposta pelo modelo foram realizados testes com variação nos parâmetros de geração da quantidade de resíduos a ser coletada e nos custos fixos e variáveis. Os resultados dos testes mostram que a rede formada pelos centros de coleta proposta pelo modelo é resiliente às variações avaliadas, sendo recomendado que os gestores considerem a possibilidade e futuras ampliações no projeto dos centros de coleta, de forma a permitir expansão de capacidade de processamento de material.

Perspectivas de pesquisas futuras incluem a investigação da dispersão dos pontos de geração no planejamento da rede reversa, tanto em relação à consideração de um número maior de pontos de coleta, quanto em relação às variações na quantidade de resíduo em cada ponto. Neste sentido, outro ponto a ser investigado é o desempenho do modelo proposto frente a um maior número de pontos de geração e de locais candidatos a centros de coleta. Ou seja, é necessário avaliar o trade-off entre a acuracidade na representação do problema real e a 'tratabilidade' computacional do modelo matemático proposto. Ainda, outras extensões para o problema planejamento de rede reversa de latas de alumínio envolvem a consideração de critérios de otimização ambientais e sociais, além dos objetivos econômicos, bem como métodos para combinação e avaliação destes critérios.

Agradecimentos. As autoras agradecem o apoio da Fundação de Amparo à Pesquisa do Estado de São Paulo - FAPESP (processo no 2013/03359-0) e da Coordenação de Aperfeiçoamento de Pessoal de Nível Superior - CAPES. 


\section{Referências}

ABAL - Associação Brasileira do Alumínio. Reciclagem no Brasil. 2016. Disponível em: http://www.abal.org.br/sustentabilidade/reciclagem/reciclagem-no-brasil/. Acesso em: $06 / 03 / 2016$.

ABRALATAS - Associação Brasileira dos Fabricantes de Latas de Alumínio. BRASIL: Vendas de latas de alumínio para bebidas e taxa de crescimento anual -1990 a 2016. 2016. Disponível em: $\quad$ http://www.abralatas.org.br/grafico/brasil-vendas-de-latas-para-bebidas-e-taxa-decrescimento-anual-1990-a-2013/. Acesso em: 02/03/2016.

ABRELPE - Associação Brasileira de Empresas de Limpeza Pública e Resíduos Especiais. Panorama dos Resíduos Sólidos no Brasil. 2016. Disponível em: http://www.abrelpe.org.br/panorama_apresentacao.cfm. Acesso em: 15/10/2016.

Agrawal, S., Singh, R. K. e Murtaza, Q. Outsourcing decisions in reverse logistics: Sustainable balanced scorecard and graph theoretic approach. Resources, Conservation and Recycling, v.108, p. 41-53, 2016.

Albareda-Sambola, M., Fernández, E. e Laporte, G. The capacity and distance constrained plant location problem. Computers \& Operations Research, v. 36, n. 2, p. 597-611, 2009.

Alshamsi, A. e Diabat, A. A reverse logistics network design. Journal of Manufacturing Systems, v. 37, Part 3, p. 589-598, 2015.

Alumur, S. A., Nickel, S., Saldanha-da-Gama, F. e Verter, V. Multi-period reverse logistics network design. European Journal of Operational Research, v. 220, n. 1, p. 67-78, 2012.

Andrade, R. M. de e Ferreira, J. A. A gestão de resíduos sólidos urbanos no Brasil frente às questões da globalização. Revista Eletrônica do Prodema-REDE, v. 6, n. 1, p. 7-22, 2011.

Cardoso, S. R., Barbosa-Póvoa, A. P., Relvas, S. e Novais, A. Q. Resilience metrics in the assessment of complex supply-chains performance operating under demand uncertainty. Omega, v. 56, p. 53-73, 2015.

CUB/m2 - Custo Unitário Básico. Indicador dos custos do setor da Construção Civil. 2016. Disponível em: http://www.cub.org.br/. Acesso em: 06/11/2016.

Dekker, R., Bloemhof, J. e Mallidis, I. Operations Research for green logistics - An overview of aspects, issues, contributions and challenges. European Journal of Operational Research, v. 219, n. 3, p. 671-679, 2012.

Ferri, G. L., Chaves, G. L. D. e Ribeiro, G. M. Reverse logistics network for municipal solid waste management: The inclusion of waste pickers as a Brazilian legal requirement. Waste Management, v. 40, p. 173-191, 2015.

Fleischmann, M., Bloemhof-Ruwaard, J. M., Dekker, R., Van der Laan, E., Van Nunen, J. A. E. E. e Van Wassenhove, L. N. Quantitative models for reverse logistics: A review. European Journal of Operational Research, v. 103, n. 1, p. 1-17, 1997.

Govindan, K., Soleimani, H. e Kannan, D. Reverse logistics and closed-loop supply chain: A comprehensive review to explore the future. European Journal of Operational Research, v. 240, n. 3, p. 603-626, 2015. 
IBGE - Instituto Brasileiro de Geografia e Estatística. Censos Demográficos. 2017. Disponível em: https://ww2.ibge.gov.br/home/estatistica/populacao/default_censo_2000.shtm. Acesso em: 20/10/2017.

Kannan, D., Diabat, A., Alrefaei, M., Govindan, K. e Yong, G. A carbon footprint based reverse logistics network design model. Resources, Conservation and Recycling, v. 67, p. 75-79, 2012.

Logožar, K., Radonjic, G. e Bastič, M. Incorporation of reverse logistics model into in-plant recycling process: A case of aluminium industry. Resources, Conservation and Recycling, v. 49, n. 1, p. 49-67, 2006.

Melo, M. T, Nickel, S. e Saldanha-da-Gama, F. Facility location and supply chain management A review. European Journal of Operational Research, v. 196, n. 2, p. 401-412, 2009.

Niknejad, A. e Petrovic, D. Optimisation of integrated reverse logistics networks with different product recovery routes. European Journal of Operational Research, v. 238, n. 1, p. 143-154, 2014.

Pishvaee, M. S., Kianfar, K. e Karimi, B. Reverse logistics network design using simulated annealing. The International Journal of Advanced Manufacturing Technology, v. 47, n. 1-4, p. 269-281, 2010.

SINAPI - Sistema Nacional de Pesquisa de Custos e Índices da Construção Civil. Custo do metro quadrado nos estados brasileiros. 2015.2 Disponível em: http://www.ibge.gov.br/home/estatistica/indicadores/precos/sinapi/sinapi_201503caderno.pdf. Acesso em: 25/06/2015.

Zhou, Y. e Wang, S. Generic model of reverse logistics network design. Journal of Transportation Systems Engineering and Information Technology, v. 8, n. 3, p. 71-78, 2008. 